\title{
Two-Dimensional Supersymmetric Microring Laser Arrays
}

\author{
Xingdu Qiao, ${ }^{1 \dagger *}$ Bikashkali Midya, ${ }^{2 \dagger}$ Zihe Gao, ${ }^{2 \dagger}$ Zhifeng Zhang, ${ }^{1}$ Haoqi Zhao, ${ }^{1}$ Tianwei Wu, ${ }^{2}$ Jieun Yim, ${ }^{2}$ \\ Ritesh Agarwal, ${ }^{2}$ Natalia M. Litchinitser, ${ }^{3}$ Liang Feng ${ }^{2,1}$ \\ ${ }^{1}$ Department of Electrical and Systems Engineering, University of Pennsylvania, Philadelphia, PA 19104, USA \\ ${ }^{2}$ Department of Materials Science and Engineering, University of Pennsylvania, Philadelphia, PA 19104, USA \\ ${ }^{3}$ Department of Electrical and Computer Engineering, Duke University, Durham, NC 27708, USA \\ ${ }^{\dagger}$ These authors contributed equally to this work. \\ *Corresponding author: xingdu@ seas.upenn.edu
}

\begin{abstract}
We develop a two-dimensional supersymmetry formalism for precise mode control. Our supersymmetric microlaser array features single-mode lasing from an evanescently coupled laser array. Our approach provides a route for designing large-scale integrated photonic systems in both classical and quantum regimes.
\end{abstract}

Keywords: Supersymmetry, laser array, micoring, single mode

\section{Introduction}

Rapid development of integrated photonics, with continuous effort to push the limit of integration density, offers a solution to future scaling of integrated photonic networks and devices. However, the wave nature of light gives rise to fundamentally inevitable mutual coupling between photonic elements closely packed in an array. Control of mutual coupling is thus the key to phase-locking of all the elements and further driving them to function collectively. Here, we demonstrate a formalism based on supersymmetry (SUSY) to enforce phase-locking and to enable coherent oscillation (I) in a twodimensional (2D) laser array of evanescently coupled microlasers.

\section{Theory}

The schematic of the proposed two-dimensional (2D) single-transverse-mode microring laser array can be described as follows: the device is composed of two parts, the five-by-five main laser array and its SUSY partners. Each ring inside the main array is designed to lase in a single longitudinal mode at around $1500 \mathrm{~nm}$. The main array, consisting of twenty-five identical aforementioned microring cavities in five columns and five rows, forms a tight binding system with evanescent coupling between nearest neighbors. Due to this proximity coupling, the originally frequency-degenerate modes of the individual cavities now form twenty-five transverse (super) modes with thirteen different eigenfrequencies. To achieve single-fundamental-transverse-mode lasing in this device, the SUSY partners are constructed such that they combined are completely isospectral with the main array except for the fundamental transverse mode. By judiciously placing the dissipative SUSY partners alongside the main array, the two systems can be coupled without heavily disturbing the main array's spectrum. Thanks to the matching of the real parts of the eigenfrequencies between the lossy SUSY partners and the main array, the suppression of all the modes except the fundamental transverse mode can be realized due to the increase of modal loss in all other modes. The fundamental mode, residing in the main array and lacking a dissipative SUSY counterpart, will be the only transverse mode that is favorable for lasing when suitable pumping is applied.

The key to the single-fundamental-mode lasing is the generation of super-partners in the 2D system. We generate the 2D SUSY partners by taking advantage of the separability between the two dimensions in the array Hamiltonian and applying second-order discrete SUSY transformation to the underlying 1D systems. The Hamiltonian of the main array can be written as 


$$
\mathrm{H}=-\sum_{m, n}\left(\kappa_{x} \alpha_{m+1, n}^{\dagger} a_{m, n}+\kappa_{y} \alpha_{m, n+1}^{\dagger} a_{m, n}+\text { h.c. }\right)
$$

where $\kappa_{x, y}$ is the nearest-neighbor coupling coefficient between adjacent rings along the $x$ and the $y$ direction respectively, $(m, n)$ labels the microring sites in the $(x, y)$ direction, $\alpha\left(\alpha^{\dagger}\right)$ is the photon creation (annihilation) operator of the resonant modes in the individual microrings, and h.c. denotes the Hermitian conjugate. Since the coupling only takes place along two perpendicular directions, i.e., either along the $x$ or the $y$ direction, the absence of diagonal coupling allows us to separate the variables in the potential profile and thus the Hamiltonian can be written in the form of a Kronecker sum

$$
\mathrm{H}=\mathrm{H}_{x} \otimes \mathrm{I}_{y}+\mathrm{I}_{x} \otimes \mathrm{H}_{y}
$$

where $\otimes$ denotes the tensor (Kronecker) product between matrices and $\mathrm{H}_{x}$ represents a one-dimensional system consisting of five coupled resonators along the $x$ direction, with coupling strength $\kappa_{x}$, while $\mathrm{I}_{y}$ is a five-by-five identity matrix representing five sites in $y$ direction without coupling in $y$. Similarly, $\mathrm{I}_{x}$ denotes the uncoupling nature in the $x$ direction, and $\mathrm{H}_{y}$ represents a one-by-five linear array with coupling strength $\kappa_{y}$ and along the $y$ direction. The problem of finding $2 \mathrm{D}$ SUSY partners thus reduces to finding the SUSY partners of $\mathrm{H}_{x}$ and $\mathrm{H}_{y}$, respectively, and then composing the 2D SUSY partners using tensor product. For the 1D SUSY problem, we rely on the second-order SUSY transformation to generate experimentally implementable partner arrays. Second-order SUSY, namely applying two consecutive SUSY transformations to the one-dimensional array, eliminates two transverse modes from the array (the fundamental mode and the highest order mode), and the resulting second-order SUSY partner only contains identical elements with the resonance frequency same as that of the main array elements. This means that second-order SUSY transformations can free the SUSY partners' elements from the typical frequency detuning resulting from a single SUSY transformation so that are experimentally favorable.

After second-order SUSY transformation on $\mathrm{H}_{x}$, we obtain the SUSY partner $\mathrm{H}_{S, x}^{(3 \times 3)}$, a three-by-three matrix representing a one-dimensional three-ring-coupled system that is isospectral to $\mathrm{H}_{x}$ except for the highest and lowest energy levels:

$$
\operatorname{spec}\left\{\mathrm{H}_{x}\right\}=\operatorname{spec}\left\{\left(\mathrm{H}_{S, x}^{(3 \times 3)} \oplus \mathrm{H}_{L, x}^{(2 \times 2)}\right)\right\}
$$

where $\oplus$ is the direct sum of two matrices and $\mathrm{H}_{L, x}^{(2 \times 2)}$ (the "leftover") is an arbitrary Hamiltonian that is isospectral to the energy levels originally in $\mathrm{H}_{x}$ but eliminated in $\mathrm{H}_{S, x}^{(3 \times 3)}$, the superscript indicates the dimension of the matrix. The spectrum of the original 2D main array Hamiltonian can now be decomposed in terms of the 1D system Hamiltonians by substituting the matrix $\mathrm{H}_{x}$ in equation (2) for its isospectral counterparts provided by equation (3):

$$
\begin{aligned}
\operatorname{spec}\{\mathrm{H}\} & =\operatorname{spec}\left\{\left(\mathrm{H}_{S, x}^{(3 \times 3)} \oplus \mathrm{H}_{L, x}^{(2 \times 2)}\right) \otimes \mathrm{I}_{y}+\left(\mathrm{I}_{x}^{(3 \times 3)} \oplus \mathrm{I}_{x}^{(2 \times 2)}\right) \otimes \mathrm{H}_{y}\right\} \\
& =\operatorname{spec}\left\{\mathrm{H}_{\text {partner }, 1}+\mathrm{H}_{L, 1}\right\}
\end{aligned}
$$

where, by taking the term $\mathrm{H}_{\text {parner, } 1} \triangleq \mathrm{H}_{S, x}^{(3 \times 3)} \otimes \mathrm{I}_{y}+\mathrm{I}_{x}^{(3 \times 3)} \otimes \mathrm{H}_{y}$, one of the main array's SUSY partners can be obtained, as indicated in the schematic. All the other SUSY partners can be acquired in a similar manner based on the expansion of the rest terms $\mathrm{H}_{L, 1} \triangleq \mathrm{H}_{L, x}^{(2 \times 2)} \otimes \mathrm{I}_{y}+\mathrm{I}_{x}^{(2 \times 2)} \otimes \mathrm{H}_{y}$, by applying second-order SUSY transformation on $\mathrm{H}_{y}$.

\section{Experiments}

The scanning electron microscope (SEM) images of the SUSY array sample fabricated on $200 \mathrm{~nm}$ thick InGaAsP multiple quantum wells are shown in Fig. 1A. The 2D SUSY microring laser array (Fig. 1A) was fabricated by electron beam lithography and reactive iron etching on the InGaAsP multiple-quantum-well platform. The gap between two rings was 200 $\mathrm{nm}$ in the main array (labeled by red dashed box) and all the SUSY partners were placed in the proximity to the main array with a gap of $330 \mathrm{~nm}$. In addition, to extract the vortex laser emission of desired topological charge from the 2D SUSY laser 
array, angular gratings were implemented along the inner sidewall of each microring. The 2D SUSY laser array was then optically pumped at the wavelength of $1064 \mathrm{~nm}$. A square pumping pattern was generated by reflecting the pumping light on a spatial light modulator (SLM). Selective pumping of the main array inside the 2D SUSY laser was then achieved by adjusting the area of the pumping pattern via SLM. The pumping pattern was further slightly defocused to improve the intensity uniformity (to reduce the speckle introduced by SLM), with most of its power shining on the main array, small but non-negligible power extending into the SUSY partners to introduce suitable loss. The emission of light from the main array inside the 2D SUSY laser was collected through an imaging system and its spectrum were measured by a monochromator, shown in Fig. 1B. The 2D SUSY array showed a spectrum with one single peak. This implies that all the other transverse modes except the fundamental mode (the mode with longest wavelength, lowest energy) were dissipated through the lossy 2D SUSY partners, and as a result the 2D SUSY laser is single-transverse-mode. The light-light curves (Fig. 1C) for the 2D SUSY microring laser shows an approximately 25 times steeper (26.3 times to be exact) slope than that of a single microring ring and a lower threshold as a result of better optical modal overlap with the gain material.. At the pumping level around 32 $\mathrm{kW} / \mathrm{cm} 2$ where the spectrum were measured, the 2D SUSY laser output power is 30 times that of a single microring laser due to the increasing of the lasing elements and the lowering of the threshold.

A

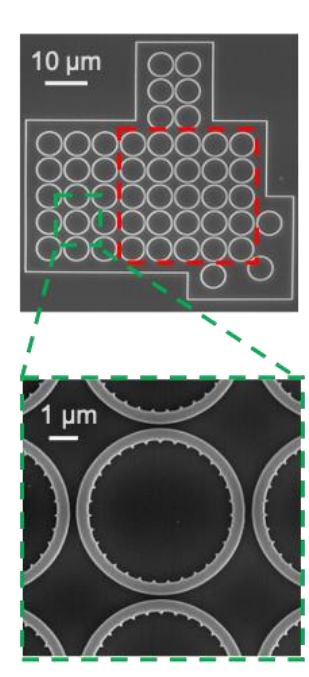

B

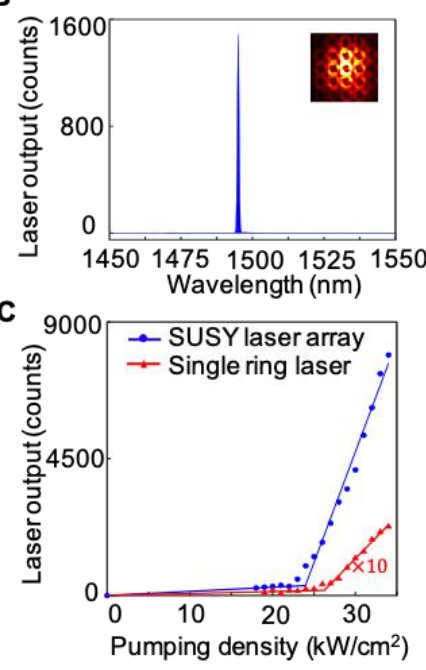

Figure 1. Experimental characterization of the higher dimensional supersymmetric microlaser array. (A) SEM images of the SUSY microlaser array. The main array is denoted by the red box, where uniform evanescent couplings are introduced with a gap of $200 \mathrm{~nm}$ between any pair of adjacent rings. The supersymmetric and auxiliary partners are placed in the proximity to the main array with a gap of $330 \mathrm{~nm}$. The zoom-in image shows detailed couplings in superpartner array 1: varying coupling strengths with a gap of $g_{x 1}=240 \mathrm{~nm}$ and the other $g_{x 2}=300 \mathrm{~nm}$ in the $x$ direction and uniform coupling equal to that in the main array with a gap of $g_{y}=200 \mathrm{~nm}$ in the $y$ direction. (B) Emission spectra, the supersymmetric microlaser array coupled with dissipative partners by selective pumping ( single

frequency lasing at $1495 \mathrm{~nm}$ ), at the pumping intensity of $32 \mathrm{~kW} / \mathrm{cm}^{2}$. The insets show the corresponding emission images at the sample plane, where intensity distribution associated with the selectively pumped SUSY laser array also confirms its operation in the

fundamental supermode. (C) Light-light curve showing the lowering of the threshold and enhancement of lasing output (the slope

efficiency) in the SUSY microlaser array compared to a single microring laser. The laser output of the single microring laser is magnified by 10 times for better visualization.

\section{Conclusion}

In summary, our 2D SUSY scheme provides a generic method to obtain single-mode high power lasing from evanescently coupled laser arrays, which is highly demanded and actively pursued for a wide range of applications, including optical communication, optical sensing, and LIDAR ranging. Bringing the SUSY scheme to 2D constitutes a powerful toolbox for potential large-scale integrated photonic systems. 


\section{References:}

[1] B. Midya, H. Zhao, X. Qiao, P. Miao, W. Walasik, Z. Zhang, N. M. Litchinitser, L. Feng, Supersymmetric microring laser arrays. Photon. Res. 7, 363-367 (2019). 